\title{
Molecular analysis of FGFR 2 and associated clinical observations in two Chinese families with Crouzon syndrome
}

\author{
YING LIN $^{1 *}$, HONGBIN GAO ${ }^{2,3^{*}}$, SIMING AI $^{1 *}$, JACOB V.P. ESWARAKUMAR ${ }^{4 *}$, TAO LI ${ }^{1}$, BINGQIAN LIU ${ }^{1}$, \\ HONGYE JIANG $^{5}$, YUHUA LIU ${ }^{1}$, XIALIN LIU ${ }^{1}$, YONGHAO LI ${ }^{1}$, YAO NI ${ }^{1}$, JIANGNA CHEN ${ }^{1}$, \\ ZHUOLING LIN ${ }^{1}$, XIAOLING LIANG ${ }^{1}$, CHENJIN JIN ${ }^{1}$, XINHUA HUANG ${ }^{1}$, LIN LU ${ }^{1}$ and YIZHI LIU ${ }^{1}$ \\ ${ }^{1}$ State Key Laboratory of Ophthalmology, Zhongshan Ophthalmic Center, Sun Yat-Sen University, Guangzhou, \\ Guangdong 510060; ${ }^{2}$ Guangdong Provincial Key Laboratory of Occupational Diseases Prevention and Treatment, \\ Guangdong Province Hospital for Occupational Diseases Prevention and Treatment, Guangzhou, Guangdong 510300; \\ ${ }^{3}$ Department of Toxicology, School of Public Health and Tropical Medicine, Southern Medical University, \\ Guangzhou, Guangdong 510515, P.R. China; ${ }^{4}$ Department of Orthopaedics and Rehabilitation, \\ Yale University School of Medicine, New Haven, CT 06520, USA; ${ }^{5}$ Department of Obstetrics and Gynecology, \\ The First Affiliated Hospital, Sun Yat-Sen University, Guangzhou, Guangdong 510000, P.R. China
}

Received July 20, 2015; Accepted May 31, 2016

DOI: $10.3892 / \mathrm{mmr} .2016 .5497$

\begin{abstract}
Crouzon syndrome, a dominantly inherited disorder and the most common type of craniosynostosis syndrome, is caused by mutations in the fibroblast growth factor receptor 2 (FGFR 2) gene, and characterized by craniosynostosis, shallow orbits, ocular proptosis, midface hypoplasia and a curved, beak-like nose. The purpose of the present study was to investigate the fibroblast growth factor receptor 2 (FGFR 2) gene in two Chinese families with Crouzon syndrome and to characterize the associated clinical features. Two families underwent complete ophthalmic examination, and three patients in two families were diagnosed with Crouzon syndrome. Genomic DNA was extracted from leukocytes of peripheral blood samples, which were collected from the family members and 200 unrelated control subjects from the same population. Exons 8 and 10 of the FGFR 2 gene were amplified using polymerase chain reaction analysis and were directly sequenced. Ophthalmic examinations, including best-corrected visual acuity, slit-lamp examination, fundus examination and Computerized Tomography scans, and physical examinations were performed to exclude systemic diseases. These patients were affected with shallow orbits
\end{abstract}

Correspondence to: Professor Lin Lu or Professor Yizhi Liu, State Key Laboratory of Ophthalmology, Zhongshan Ophthalmic Center, Sun Yat-Sen University, 54 Xianlie Road, Guangzhou, Guangdong 510060, P.R. China

E-mail:drlulin@126.com

E-mail: yzliu62@yahoo.com

*Contributed equally

Key words: Crouzon syndrome, fibroblast growth factor receptor 2 gene, mutation and ocular proptosis, accompanied by midface hypoplasia, craniosynostosis, strabismus or papilloedema, with clinically normal hands and feet. A heterozygous FGFR 2 missense mutation, c.811-812insGAG (p.273insGlu) in exon 8 was identified in the affected individual, but not in the unaffected family members or the normal control individuals in family 1. In family 2, another heterozygous FGFR 2 missense mutation, c.842A $>$ G (P.Tyr281Cys or Y281C), in exon 8 was identified in the affected boy and his mother, but not in the unaffected family members or the normal control individuals. Although FGFR 2 gene mutations and polymorphisms have been reported in various ethnic groups, particularly in the area of osteology, the present study reported for the first time, to the best of our knowledge, the identification of two novel FGFR 2 gene mutations in Chinese patients with Crouzon syndrome.

\section{Introduction}

Crouzon syndrome, a type of craniosynostosis, is a dominantly inherited disorder, which is predominantly caused by mutations in the fibroblast growth factor receptor 2 (FGFR 2) gene and is characterized by craniosynostosis, shallow orbits, ocular proptosis, midface hypoplasia and a curved, beak-like nose (1-4).

The most common genetic mutations of FGFR 2, located at chromosome 10q26, are localized at exons IIIa (exon 8) and IIIc (exon 10) that encode the extracellular immunoglobulin-like III (IgIII) domain of the protein (5-7).

Although Crouzon syndrome is inherited as an autosomal dominant trait, several cases present as de novo mutations arising from unaffected parents (8-11). The present study reported the mutational analyses of three patients with Crouzon syndrome from separate families at the gene level and reported its associated clinical features, resulting in the identification of two heterozygous mutations. 


\section{Patients and methods}

Crouzon syndrome families. Two probands in two Chinese families were diagnosed as having Crouzon syndrome at the Zhongshan Ophthalmic Center (Guangzhou, China). The proband of family 1 (Fig. 1) was a one-year-old girl and was the second child of healthy unrelated parents, vaginally delivered maturely at 39 weeks. The second proband, in family 2 , (Fig. 2) was a three-year-old boy and was also the second child of his family. For the present study, ophthalmic examinations of these two families were performed, as follows: Visual acuity was examined using the Early Treatment Diabetic Retinopathy Study chart (Precision Vision, LaSalle, IL, USA). Anterior segment images were captured using a BX 900 Slit Lamp (Haag-StreitAG, Köniz, Switzerland). Anterior segment measurements were obtained using Pentacam ${ }^{\circledR}$ HR version 70700 (OCULUS Optikgeräte GmbH, Wetzlar, Germany). Fundus imaging was performed using a Heidelberg Retina Angiograph (Heidelberg Engineering $\mathrm{GmbH}$, Heidelberg, Germany). In addition, physical examinations, including blood examination, a urine test, electrocardiogram, chest X-ray, blood biochemistry test, blood lipid and blood coagulation tests, were performed to exclude systemic diseases. The study was approved by the ethics committee of Zhongshan Ophthalmic Center, Sun Yat-Sen University.

Sample collection. The affected families were identified at Zhongshan Ophthalmic Center. In addition, 200 subjects from the same population, but without diagnostic features of Crouzon syndrome, were recruited to serve as normal controls. Informed consent was obtained from all participating individuals and, in accordance with the principles of the Declaration of Helsinki, venous blood samples were collected from the two families and 200 controls for genomic DNA extraction from peripheral blood leukocytes, using the a DNA extraction kit (Qiagen, Inc., Valencia, CA, USA) with standard protocols.

Mutation detection. Exons 8 and 10 of the FGFR 2 gene were amplified using polymerase chain reaction (PCR) analysis with the following primers: FGFR2-8 (IIIa), forward 5'-GGT CTCTCATTCTCCCATCCC-3', reverse 5'-CCAACAGGA AATCAAAGA ACC-3' (product size, 325 bp); FGFR2-10 (IIIc), forward 5'-CCTCCACAATCATTCCTGTGTC-3', reverse 5'-ATAGCAGTCAACCAAGAAAAGGG-3' (product size, 257 bp) (9-10) (Beijing Genomics Institute, Guangzhou, China). Briefly, PCR was performed with a $50 \mu 1$ reaction volume with $2 \mu \mathrm{l}$ each primer, $2 \mu \mathrm{l}$ DNA, $25 \mu \mathrm{l}$ buffer mix and $19 \mu \mathrm{H}_{2} \mathrm{O}$. All reagents used for PCR were purchased from (Takara Bio, Inc., Tokyo, Japan).

The cycling profile included one cycle at $94^{\circ} \mathrm{C}$ for $5 \mathrm{~min}$, followed by 40 cycles at $94^{\circ} \mathrm{C}$ for $45 \mathrm{sec}, 61^{\circ} \mathrm{C}$ for $45 \mathrm{sec}$, and $72^{\circ} \mathrm{C}$ for $45 \mathrm{sec}$, with one cycle at $72^{\circ} \mathrm{C}$ for $10 \mathrm{~min}$. The PCR products were sequenced from both directions using an ABI3730 automated sequencer (PE Biosystems, Foster City, CA, USA). The sequencing results were analyzed using Chromas (version 2.3; Technelysium Pty., Ltd., Brisbane, QLD, Australia), and they were compared with the reference sequences in the database at the National Center for Biotechnology Information (NCBI; NC_000010).

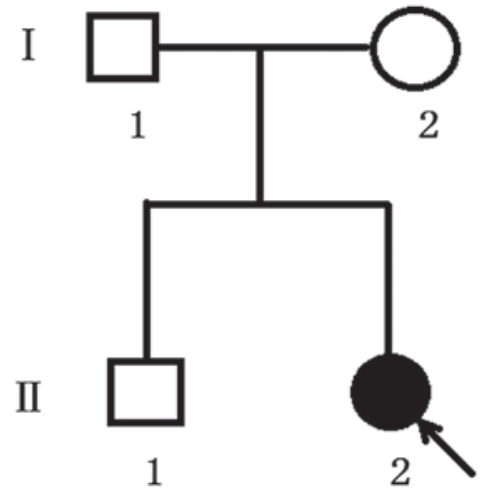

Figure 1. Pedigree of a Chinese family (family 1) with Crouzon syndrome. Squares denote males, circles denote females. Shaded symbol indicates ophthalmologist-confirmed Crouzon syndrome. Arrow indicates the proband.

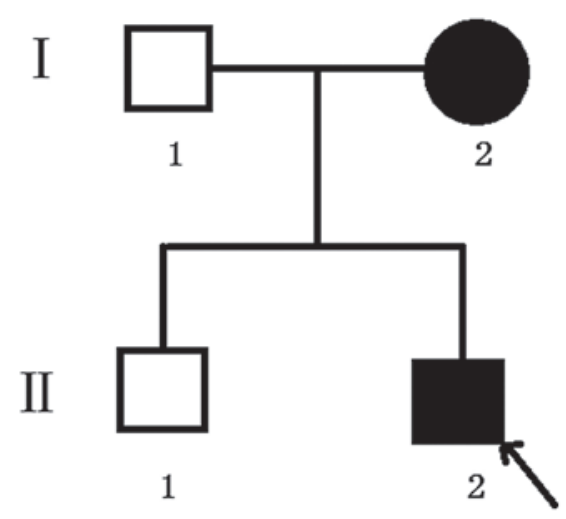

Figure 2. Pedigree of a Chinese family (family 2) with Crouzon syndrome. Squares denote males, circles denote females. The shaded symbols indicate ophthalmologist-confirmed Crouzon syndrome. The arrow indicates the proband.

A

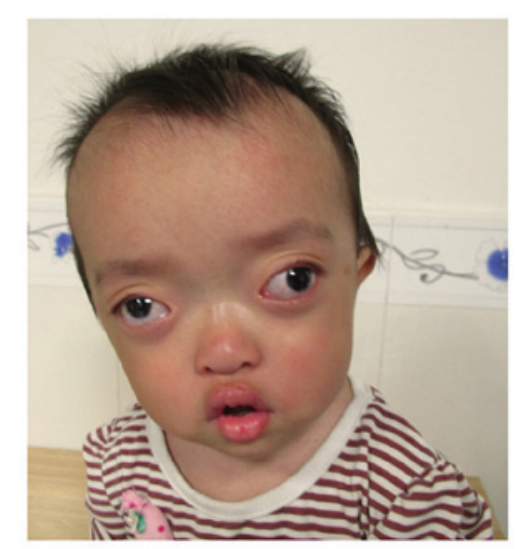

B

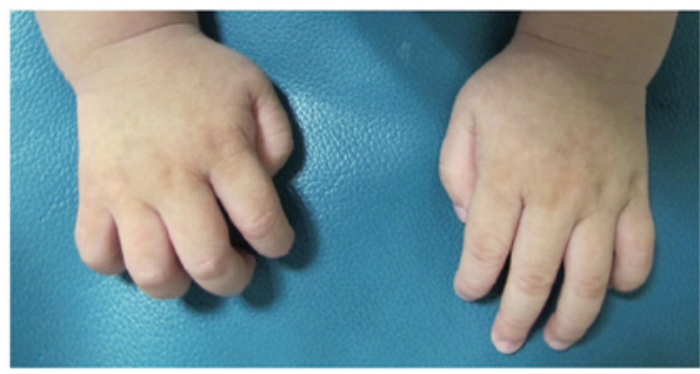

Figure 3. Images of the proband of family 1. (A) Proband of family 1 exhibited ocular proptosis, strabismus and midface hypoplasia. (B) Clinical image of the hands of the proband of family 1 . 

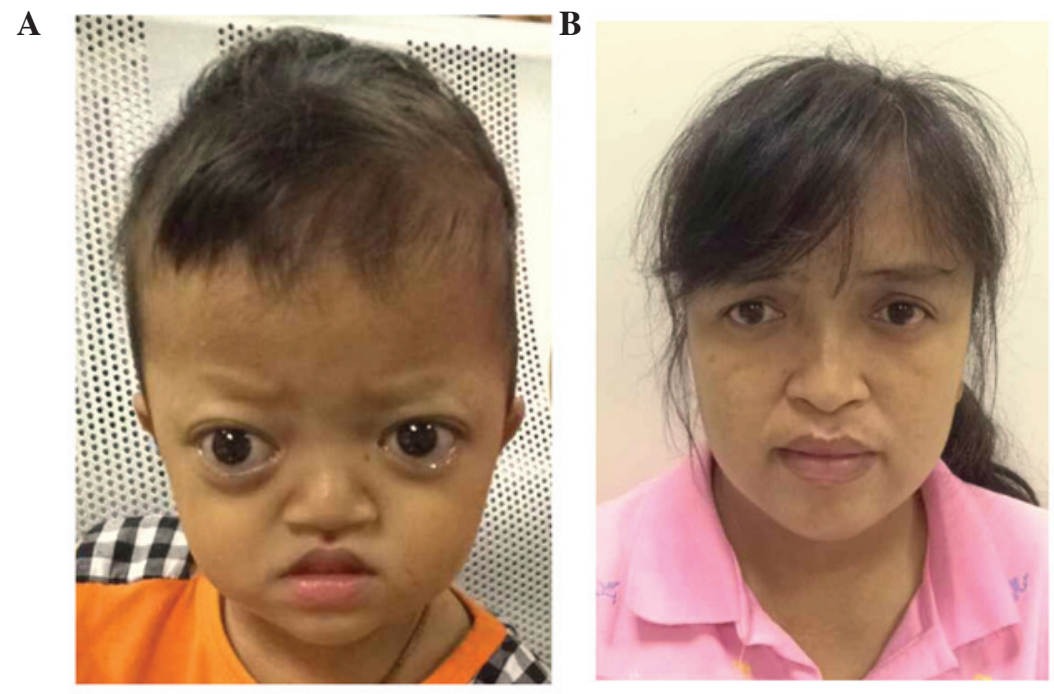

C
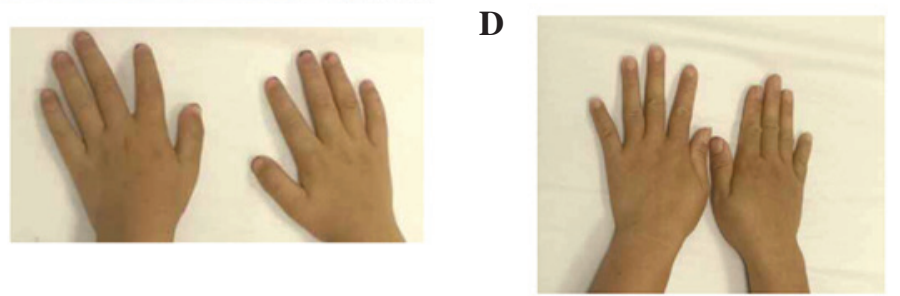

Figure 4. Images of the proband of family 2 and his mother. (A) Proband of family 2 exhibited ocular proptosis and midface hypoplasia. (B) Mother of the proband of family 2 , who presented with a similar appearance to her son. (C) Clinically normal hands of the proband of family 2. (D) Clinically normal hands of the mother of the proband of family 2.

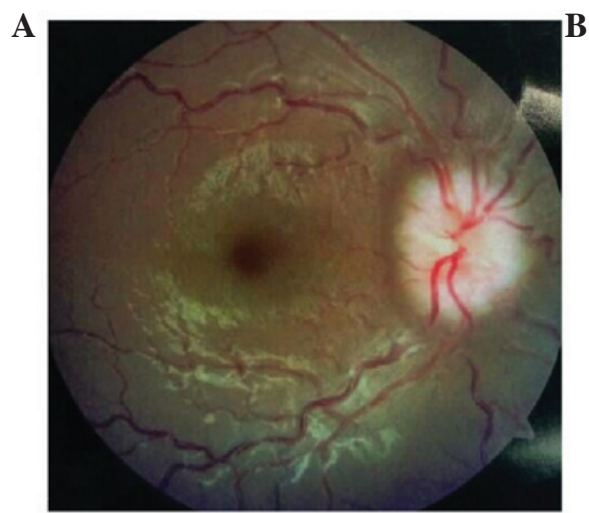

OD

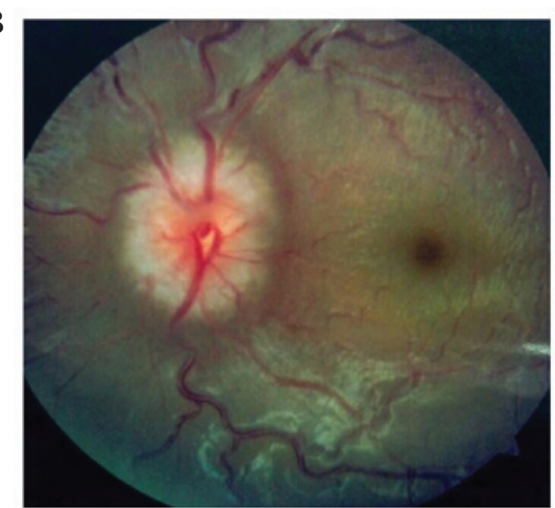

OS

C

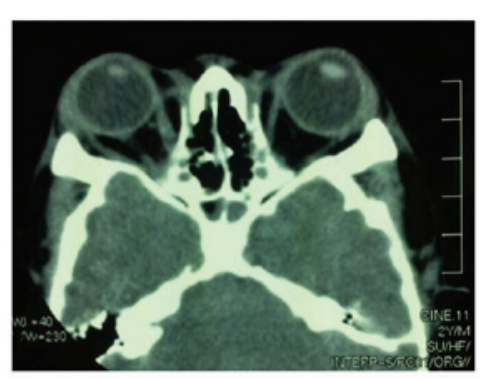

Figure 5. Images of the examination of the proband of family 2. (A and B) Papilloedema of both eyes were noted. (C) Shallow orbits were found on Computed Tomography examination. OD, right eye; OS, left eye.

\section{Results}

Clinical data. The Chinese families included in the present study were from the southern area of China. In two successive generations, one individual was found to have a congenital disease. The proband of family 1 was referred by her local pediatrician at the age of 2 months, owing to concerns about her elongated head shape and a possible diagnosis of sagittal 


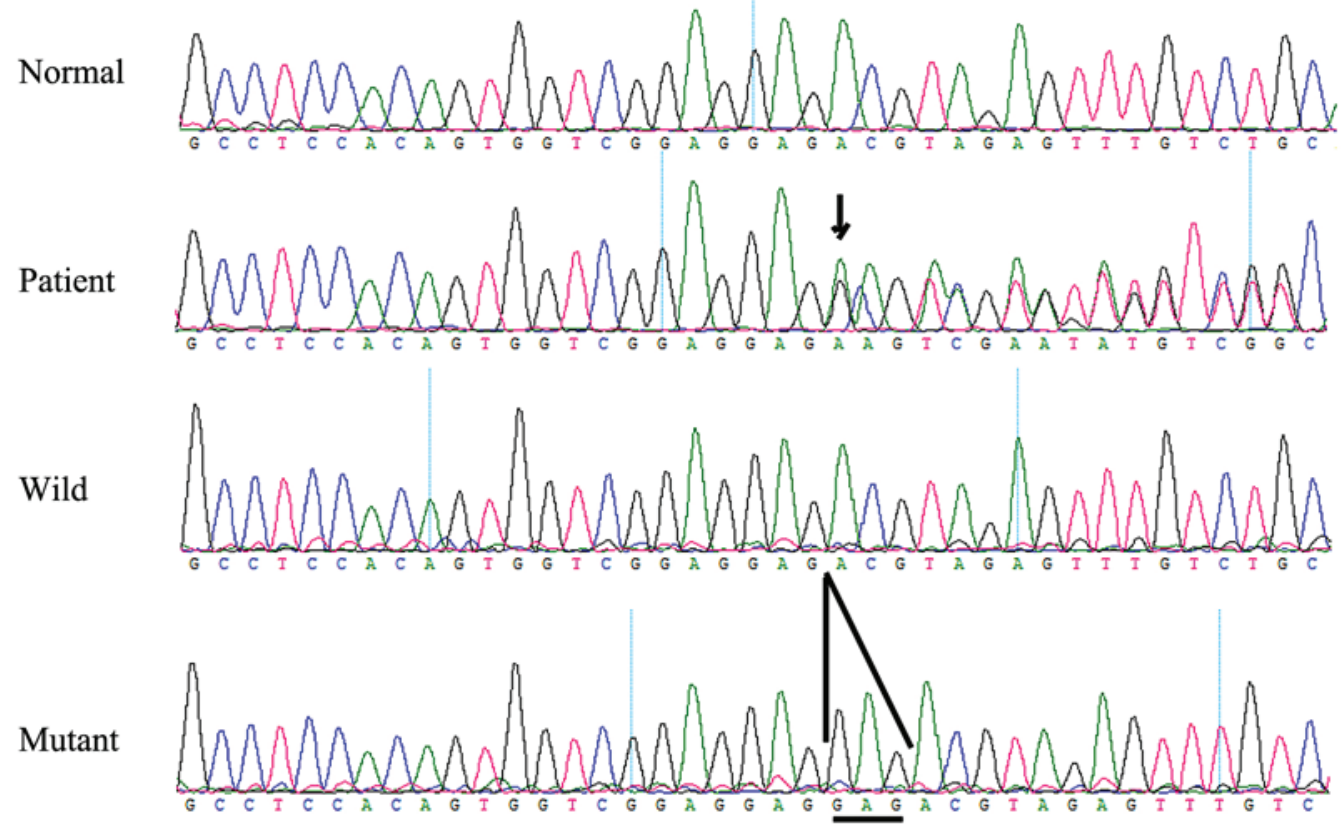

Figure 6. DNA sequence of a region of the $F G F R 2$ gene in the affected and unaffected individuals of family 1 . A heterozygous $F G F R 2$ missense mutation, c.811-812insGAG (p.273insGlu), in exon 8 was identified in the affected individual, but not in any of the unaffected family members or the normal controls The mutation caused the insertion of glutamic acid in the position of 273 of FGFR 2. Patient; c.811-812insGAG (p.273insGlu) mutation in exon 8 in the affected individuals; wild, sequence of the normal allele of exon 8 subcloned into the pGEM-T vector (used as a control); mutant, heterozygous missense mutation, c.811-812insGAG (p.273insGlu), in exon 8 in the affected individuals. This mutation caused the insertion of glutamic acid in position of 273 of the FGFR 2 gene. FGFR 2, fibroblast growth factor receptor 2.
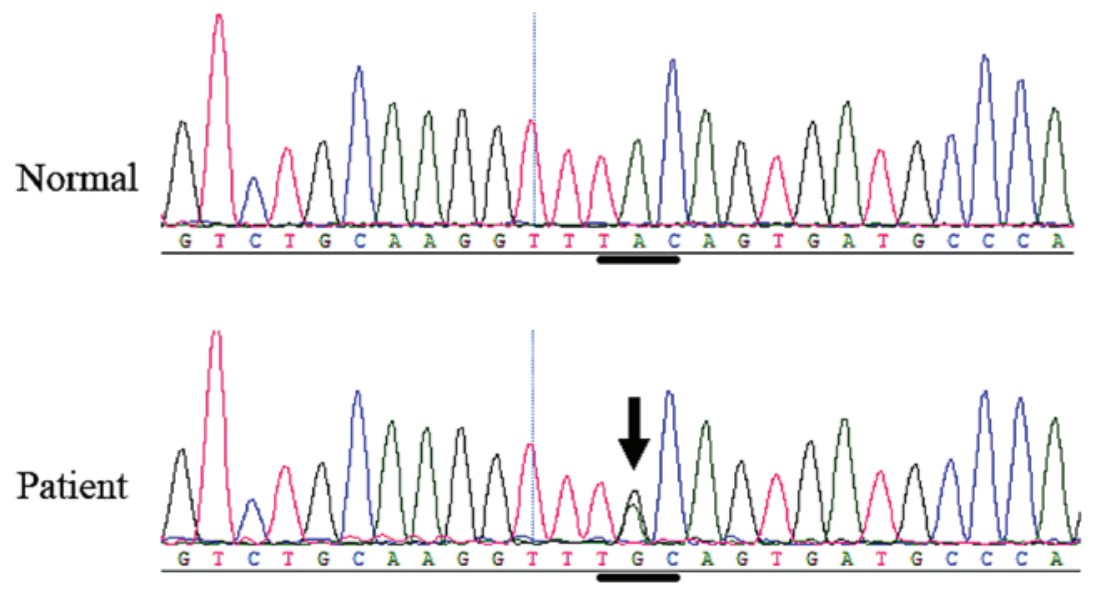

Figure 7. DNA sequence of the reigon of the FGFR 2 gene in the affected and unaffected individuals of family 2 . In family 2 , the heterozygous $F G F R 2$ missense mutation, c.842A $>$ G (P.Tyr281Cys or Y281C), in exon 8 was identified in the affected boy and his mother, but not in any of the unaffected family members or the normal control individuals. The mutation caused the tyrosine 281 codon to change to a cysteine codon (arrow). FGFR 2, fibroblast growth factor receptor 2 .

synostosis. The proband had otherwise been developing well with normal feeding and steady weight gain following birth. There was no history of learning difficulties or genetic problems in the family. The parents considered her development to be equivalent to her age-matched peers.

On examination, the proband exhibited shallow orbits and ocular proptosis, accompanied by midface hypoplasia, craniosynostosis and a curved, beak-like nose (Fig. 3A), with clinically normal hands and feet (Fig. 3B). The proband also showed exotropia of the left eye, although the corneas were normal in size and transparency, and the lenses were positioned normally and remained clear. As the child was just 1 year old, it was not possible to assess visual acuity, however, visual tracking was present. The parents and the old brother of the proband showed normal visual acuity and eye examinations.

The proband of family 2 had midface hypoplasia and craniosynostosis (Fig. 4A). His mother's condition was less severe, with normal visual acuity (Fig. 4B). The proband and his mother had clinically normal hands and feet (Fig. 4C and D). The proband had papilloedema of both eyes (Fig. 5A and B) and had a history of intracranial hypertension (1 year previously). The Computed Tomography examination revealed 
shallow orbits (Fig. 5C). No abnormalities were detected in the corneas or lens.

Mutation screening. A heterozygous FGFR 2 missense mutation, c.811-812insGAG (p.273insGlu), in exon 8 (Fig. 6) was identified in the affected individual, but was not identified in any of the unaffected family members or the normal control individuals. This mutation causes the insertion of glutamic acid in position of 273 of the FGFR 2 gene. In family 2, another heterozygous FGFR 2 missense mutation, c.842A $>\mathrm{G}$ (P.Tyr281Cys or Y281C), in exon 8 (Fig. 7) was identified in the affected boy and his mother, but was not identified in any of the unaffected family members or the normal control individuals. The mutation causes the tyrosine 281 codon to change to a cysteine codon.

\section{Discussion}

In the present study, two mutations in exon 8 of the FGFR 2 gene were identified, which were associated with Crouzon syndrome: A de novo mutation, c.811-812insGAG (p.273insGlu), in family 1 and a familial mutation, c.842A>G (p.Tyr281Cys or Y281C), in family 2 . These mutations, rather than representing a rare polymorphism in the normal population, were the causative mutations in these two families.

The c.811-812insGAG (p.273insGlu) mutation was identified for the first time in the FGFR 2 gene in Chinese patients, and, to the best of our knowledge, has not previously been reported either in China or elsewhere. However, the c.842A $>$ G (P.Tyr281Cys or Y281C) mutation, identified in family 2, has been previously reported outside of China (12). Although Crouzon syndrome is inherited as an autosomal dominant trait, several cases are sporadic and present as de novo mutations arising from unaffected parents, as observed in family 1 in the present study.

The most common genetic mutation of FGFR 2 has been localized in the third Ig-like domain and in the flanking linker regions, coded by exons IIIa (exon 8) and IIIc (exon 10) $(13,14)$. The two mutations found in the two families in the present study were in exon IIIa. The mutations may disrupt the intra-Ig domain disulfide bond, thus leading to changes in FGF signaling, and may induce the activation or downregulation of FGFR 2 (15-20).

Okajima et al (21) reported that certain patients with an FGFR 2 mutation may have Peters anomaly, optic nerve hypoplasia, scleralization of the cornea and corectopia in craniosynostosis syndromes. In the present study, the proband of family 2 had papilloedema, which expands the clinical manifestations of Crouzon syndrome.

In conclusion, the present study identified two mutations of FGFR 2 in two Chinese families with Crouzon syndrome. This finding expands the mutation spectrum of FGFR 2, and is useful and valuable for genetic counseling and prenatal diagnosis in families with Crouzon syndrome with ocular disorders.

\section{Acknowledgements}

This study was supported by the National Natural Science Foundation of China (grant no. 81500709), the Project of Fundamental Research Funds of State Key Laboratory of Ophthalmology (grant nos. 2011Q09, 2012KF03 and 2014QN04), and the Medical Scientific Research Foundation of Guangdong Province (grant no. A201646).

\section{References}

1. Reardon W, Winter RM, Rutland P, Pulleyn LJ, Jones BM and Malcolm S: Mutations in the fibroblast growth factor receptor 2 gene cause Crouzon syndrome. Nat Genet 8: 98-103, 1994.

2. Gorry MC, Preston RA, White GJ, Zhang Y, Singhal VK, Losken HW, Parker MG, Nwokoro NA, Post JC and Ehrlich GD: Crouzon syndrome: Mutations in two spliceoforms of FGFR2 and a common point mutation shared with Jackson-Weiss syndrome. Hum Mol Genet 4: 1387-1390, 1995.

3. Oldridge M, Lunt PW, Zackai EH, McDonald-McGinn DM, Muenke M, Moloney DM, Twigg SR, Heath JK, Howard TD, Hoganson G, et al: Genotype-phenotype correlation for nucleotide substitutions in the IgII-IgIII linker of FGFR2. Hum Mol Genet 6: 137-143, 1997.

4. Murano I: Crouzon syndrome. Nihon Rinsho (Suppl 3): S416-S417, 2006 (In Japanese).

5. Steinberger D, Reinhartz T, Unsöld R and Müller U: FGFR2 mutation in clinically nonclassifiable autosomal dominant craniosynostosis with pronounced phenotypic variation. Am J Med Genet 66: 81-86, 1996.

6. Park WJ, Meyers GA, Li X, Theda C, Day D, Orlow SJ, Jones MC and Jabs EW: Novel FGFR2 mutations in Crouzon and Jackson-Weiss syndromes show allelic heterogeneity and phenotypic variability. Hum Mol Genet 4: 1229-1233, 1995.

7. Meyers GA, Day D, Goldberg R, Daentl DL, Przylepa KA, Abrams LJ, Graham JM Jr, Feingold M, Moeschler JB, Rawnsley E, et al: FGFR2 exon IIIa and IIIc mutations in Crouzon, Jackson-Weiss and Pfeiffer syndromes: Evidence for missense changes, insertions, and a deletion due to alternative RNA splicing. Am J Hum Genet 58: 491-498, 1996.

8. Steinberger D, Collmann H, Schmalenberger B and Müller U: A novel mutation $(\mathrm{a} 886 \mathrm{~g})$ in exon 5 of FGFR2 in members of a family with Crouzon phenotype and plagiocephaly. J Med Genet 34: 420-422, 1997.

9. Lin Y, Ai S, Chen C, Liu X, Luo L, Ye S, Liang X, Zhu Y, Yang $\mathrm{H}$ and Liu Y: Ala344Pro mutation in the FGFR2 gene and related clinical findings in one Chinese family with Crouzon syndrome. Mol Vis 18: 1278-1282, 2012.

10. Lin Y, Liang X, Ai S, Chen C, Liu X, Luo L, Ye S, Li B, Liu Y and Yang H: FGFR2 molecular analysis and related clinical findings in one Chinese family with Crouzon syndrome. Mol Vis 18: 449-454, 2012.

11. Hollway GE, Suthers GK, Haan EA, Thompson E, David DJ, Gecz J and Mulley JC: Mutation detection in FGFR2 craniosynostosis syndromes. Hum Genet 99: 251-255, 1997.

12. Kan SH, Elanko N, Johnson D, Cornejo-Roldan L, Cook J, Reich EW, Tomkins S, Verloes A, Twigg SR, Rannan-Eliya S, et al: Genomic screening of fibroblast growth-factor receptor 2 reveals a wide spectrum of mutations in patients with syndromic craniosynostosis. Am J Hum Genet 70: 472-486, 2002.

13. Tartaglia M, Valeri S, Velardi F, Di Rocco C and Battaglia PA: Trp290Cys mutation in exon IIIa of the fibroblast growth factor receptor 2 (FGFR2) gene is associated with Pfeiffer syndrome. Hum Genet 99: 602-606, 1997.

14. Ke R, Yang X, Tianyi C, Ge M, Lei J and Mu X: The C342R mutation in FGFR2 causes Crouzon syndrome with elbow deformity. J Craniofac Surg 26: 584-586, 2015.

15. Padmanabhan V, Hegde AM and Rai K: Crouzon's syndrome: A review of literature and case report. Contemp Clin Dent 2: 211-214, 2011.

16. Robin NH, Falk MJ and Haldeman-Englert CR: FGFR-Related Craniosynostosis Syndromes. In: Pagon RA, Adam MP, Ardinger HH, Wallace SE, Amemiya A, Bean LJH, Bird TD, Fong CT, Mefford HC, Smith RJH and Stephens K (eds). GeneReviews ${ }^{\circledR}$ (http://www.ncbi.nlm.nih.gov/books/ NBK1455/). University of Washington, Seattle, WA, 1993.

17. Snyder-Warwick AK, Perlyn CA, Pan J, Yu K, Zhang L and Ornitz DM: Analysis of a gain-of-function FGFR2 Crouzon mutation provides evidence of loss of function activity in the etiology of cleft palate. Proc Natl Acad Sci USA 107: 2515-2520, 2010.

18. Piccione M, Antona V, Niceta M, Fabiano C, Martines M, Bianchi A and Corsello G: Q289P mutation in the FGFR2 gene: First report in a patient with type 1 Pfeiffer syndrome. Eur J Pediatr 168: 1135-1139, 2009. 
19. Lapunzina P, Fernández A, Sánchez Romero JM, Delicado A, Sáenz de Pipaon M, López Pajares I and Molano J: A novel insertion in the FGFR2 gene in a patient with Crouzon phenotype and sacrococcygeal tail. Birth Defects Res A Clin Mol Teratol 73: 61-64, 2005.

20. Gong SG: The Fgfr2 W290R mouse model of Crouzon syndrome. Childs Nerv Syst 28: 1495-1503, 2012.
21. Okajima K, Robinson LK, Hart MA, Abuelo DN, Cowan LS, Hasegawa T, Maumenee IH and Jabs EW: Ocular anterior chamber dysgenesis in craniosynostosis syndromes with a fibroblast growth factor receptor 2 mutation. Am J Med Genet 85: 160-170, 1999. 